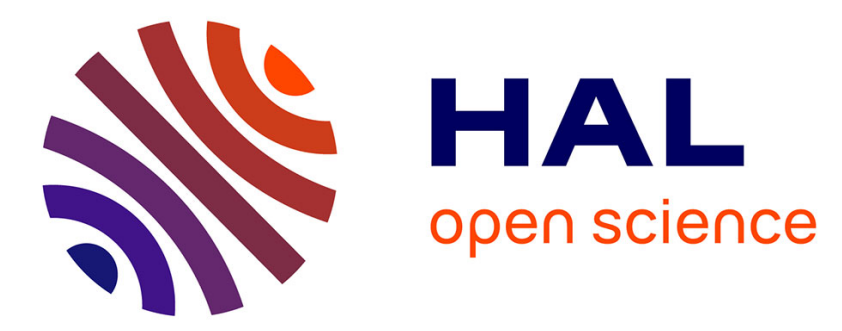

\title{
Identification of very large scale structures in the boundary layer over large roughness elements
}

\author{
Laurent Perret, Franck Kerhervé
}

\section{To cite this version:}

Laurent Perret, Franck Kerhervé. Identification of very large scale structures in the boundary layer over large roughness elements. Experiments in Fluids, 2019, 60 (6), pp.97. 10.1007/s00348-019-27497. hal-02285149

\author{
HAL Id: hal-02285149 \\ https://hal.science/hal-02285149
}

Submitted on 10 May 2021

HAL is a multi-disciplinary open access archive for the deposit and dissemination of scientific research documents, whether they are published or not. The documents may come from teaching and research institutions in France or abroad, or from public or private research centers.
L'archive ouverte pluridisciplinaire HAL, est destinée au dépôt et à la diffusion de documents scientifiques de niveau recherche, publiés ou non, émanant des établissements d'enseignement et de recherche français ou étrangers, des laboratoires publics ou privés. 


\title{
Identification of very large scale structures in the boundary layer over large roughness elements
}

\author{
Laurent Perret $^{1} \cdot$ Franck Kerhervé ${ }^{2}$
}

\begin{abstract}
The dynamics of a turbulent boundary layer developing over a cube array is investigated based on stereoscopic PIV measurements performed in a boundary layer wind tunnel. The fluctuating streamwise velocity component $u^{\prime}$ is analyzed via the recently introduced Spectral Proper Orthogonal Decomposition method (S-POD). A modification of the definition of the spatial S-POD modes is proposed so that they are orthonormal to each other. This results in spatio-temporal modes which reflect the statistical organization of the most energetic structures both in space and time. It is first demonstrated that the first S-POD modes of $u^{\prime}$ correspond to large-scale elongated coherent structures of low or high momentum. The existence of very large scale structures whose length spans several boundary layer thickness is demonstrated, in agreement with the recent similar findings for flows over smooth wall. These structures are found to be non-negligible contributors to the Reynolds shear stress and the turbulent kinetic energy. Their relationship with the smaller scales of the flow is investigated via the computation of joint third-order statistics and is shown to be of non-linear nature.
\end{abstract}

\section{Introduction}

During the past few years, very large scale motions (VLSMs) in turbulent boundary layers over smooth walls have received renewed attention from the research community. Both numerical and experimental studies have highlighted their influence on the near-wall turbulence and their contribution to the turbulent kinetic energy and Reynolds shear stress in different types of wall-bounded flows such as pipe flows (Monty et al. 2007), channel flows (del Alamo and Jimenez 2003), laboratory boundary layers (Marusic and
Hutchins 2008) and atmospheric boundary layers (Guala et al. 2011). Common features of the VLSMs found in wallbounded flows are that they consist of elongated low- and high-speed regions relative to the mean flow populating the logarithmic and outer layers (Hutchins and Marusic 2007). Their streamwise extent scales with boundary layer thickness $\delta$ and can reach several times $\delta$ (Guala et al. 2011). They were shown to be animated by a meandering motion in the horizontal plane (Hutchins and Marusic 2007). Mathis et al. (2009) demonstrated that they interact with near-wall turbulence through an amplitude modulation mechanism. The

Laurent Perret

laurent.perret@ec-nantes.fr

1 Centrale Nantes, LHEEA, UMR 6598 CNRS, Nantes, 1 rue de la Noë, BP 92101, 44321 Nantes Cedex 3, France

2 Institut PPRIME, Université de Poitiers - ENSMA, UPR 6609 CNRS, Poitiers, France 
finding of this last characteristic relies on the clear spectral separation between large-scale motions and the near-wall turbulence found in high-Reynolds number flows (Guala et al. 2011; Mathis et al. 2009).

At the same time, attention has been devoted to the structure of boundary layer flows developing over rough walls, at laboratory scales (see Jimenez 2004 for a review) or in atmospheric flows over urban or vegetation canopies (Blackman and Perret 2016; Finnigan 2000; Hutchins et al. 2012; Inagaki and Kanda 2010; Perret et al. 2019; Takimoto et al. 2011), demonstrating similarities between flows over smooth and rough walls. In particular, the presence of streaky patterns of low- and high-speed regions, of ejection and sweep motions associated with the hairpin model (Adrian 2007), and the organization of hairpin vortices in packets have been evidenced. Recently, Inagaki and Kanda (2010) showed the presence of very large scale elongated low-speed regions with some sub-structures included in these streaks in the atmospheric flow developing over an array of cubes. These structures, identified by filtering the time series in the spanwise direction from a spanwise array of 15 sonic anemometers, share some common features with the VLSMs evidenced in smoothwall flows. For an atmospheric boundary layer developing over a cubical array arranged in a square pattern, Takimoto et al. (2011) showed the intermittent presence of large-scale upward motions across the whole wall-normal cross section between two cubes inside the canopy. These were found to be correlated with the presence of low-speed streaks in the boundary layer. A similar organization was observed by Perret and Savory (2013) for a turbulent boundary layer over a street-canyon model. From their particle image velocimetry (PIV) measurements performed over a cube array, Rivet et al. (2012) evidenced the presence of vortex clusters which were intermittently shed off the roughness elements and transported in the boundary layer. They also showed that the dynamics of the associated shear layers developing from the top of the obstacles is correlated with the occurrence of low- and high-speed events in the log region. For the same configuration, using a multi-time delay stochastic estimation technique applied to PIV data in the near-wall region synchronized with hotwire anemometry (HWA) velocity measurements in the logarithmic layer, Blackman and Perret (2016) succeeded in separating the large-scale contribution of the overlying boundary layer from the flow near the top of the obstacles. Their analysis of the scale-decomposed skewness of the streamwise velocity component demonstrated the nonlinear nature of the interaction between these two components of the flow. They evidenced a multi-scale interaction mechanism that resembles the amplitude modulation one found by Mathis et al. (2009) in boundary layers over smooth wall, in agreement with the recent findings obtained by Anderson (2016) from large-eddy simulations of flows over cubical roughness. These findings have been extended by Blackman et al. (2018b) to street canyons of different width, with various upstream roughness configuration of urban type. Placidi and Ganapathisubramani (2015) recently performed a snapshot-based proper orthogonal decomposition (POD) of PIV measurements obtained in a boundary layer developing over urban-like roughness elements. They explored the spatial characteristics and the behavior of the flow and its dependence on wall morphology. The most energetic modes, containing about $50 \%$ of the turbulent kinetic energy, were found to consist of large-scale elongated regions of low and high momentum, whose characteristics proved to be independent of the wall configuration. The results obtained in flows over rough walls suggest that, in spite of the strong disturbance of the flow at the wall, VLSMs exist and interact with the canopy flow in a similar manner as in smooth-wall boundary layers. However, sharp edge bluff bodies such as those encountered in urban canopies generate coherent structures of scales much larger than that existing in the near-wall region of a smooth-wall boundary layer (Volino et al. 2011; Perret et al. 2019). Therefore, the clear spectral separation between large-scale motions and the near-wall turbulence found in high-Reynolds number smooth-wall boundary layers may not exist in boundary layer developing of arrays of bluff bodies. As pointed by Takimoto et al. (2011), the knowledge of the connections between the canopy flow and organized structures in the boundary layer is currently still limited.

When aiming at investigating the presence of large-scale structures in a turbulent boundary layer developing over a cubical roughness array and studying their dynamical link with the canopy flow, two challenges then arise. First, the clear spectral separation between the near-wall turbulence and the energetic scales in the logarithmic region found in smooth-wall turbulent flows is not expected to exist anymore. In turbulent boundary layers over rough walls in cases for which the flow is in fully-rough regime, the mean streamwise velocity component reads, when written in its meteorological form (Castro 2007),

$\frac{\bar{u}(z)}{u_{\tau}}=\frac{1}{\kappa} \log \left(\frac{z-d}{z_{0}}\right)$,

where $\bar{u}(z)$ is the mean streamwise velocity component at height $z, u_{\tau}$ the friction velocity, $\kappa=0.4$ the von Karman constant, $d$ the displacement height and $z_{0}$ the roughness length, equivalent of the streamwise velocity component deficit used in the engineering community. In the fully rough regime, the ratio $z_{0} / h$ depends only on the roughness geometry and not on the Reynolds number anymore. In urban environment, a typical value of the ratio $z_{0} / h$ is 0.1 while 
the ratio $\delta / h$ is comprised between 10 and 20 (Grimmond and Oke 1999; Jimenez 2004). With these values and by extrapolating the $\log$-law up to $z=\delta$, the ratio between typical temporal scales in the boundary layer and close to the canopy, $T_{\delta}=\delta / U_{\delta}$ and $T_{h}=h / u_{\tau}$ (where $U_{\delta}$ is the integral velocity scale related to the largest scales, and $u_{\tau}$ the friction velocity), respectively, can be estimated as being close to unity (Perret et al. 2019):

$\frac{T_{\delta}}{T_{h}}=\frac{\delta}{h} \frac{u_{\tau}}{U_{\delta}}=\frac{\delta}{h}\left[\frac{1}{\kappa} \log \left(\frac{\delta}{h} \frac{h}{z_{0}}\right)\right]^{-1}$.

A lack of separation in the spectral domain between the most energetic scales from the boundary layer and those existing near the canopy thus appears to be an intrinsic characteristic of boundary layers developing over urban terrains and can, therefore, be expected in small-scale wind tunnel experiments if the flow is correctly scaled (i.e. similarity between model and full scale of $\delta / h$ and the Jensen number $z_{0} / h$ must be ensured, Savory et al. 2013). The second challenge arising is an experimental one. Performing reliable measurements of time series of the velocity via multi-component hot-wire anemometry can turn out to be difficult and even impossible due to the high local turbulent intensities and the highly three-dimensional character of the flow close to the canopy top (Perret and Rivet 2018). To circumvent this issue, optical velocimetry methods have been recently employed in flows over urban-like roughness arrays. Herpin et al. (2018) succeeded in performing laser Doppler anemometry (LDA) measurements within the canopy with a sampling rate high enough to allow for the computation of velocity spectra but also cross-spectra between the LDA measurements and the streamwise velocity component measured by HWA in the outer region. Basley et al. (2018) performed stereoscopic PIV in a wall-parallel plane just above the canopy top. The streamwise extent of their PIV data was large enough to allow for the reconstruction of the large-scale motions using Taylor's hypothesis.

To investigate the interaction between boundary layer and canopy scales, an alternative scale-separating method which can capture the spatial heterogeneities of the flow in the canopy region is proposed. This methods differs from the one now classically used in the literature (Mathis et al. 2009) in that it does not rely on the use of a spectral cutoff filter nor require the use of two-point time-resolved velocity measurements (such as the similar LSE-based methods employed by Blackman and Perret 2016). To circumvent the two above-mentioned issues, the fluctuating streamwise velocity component, measured by low-speed stereoscopic $\mathrm{PIV}$, is analyzed via the use of a modified version of the now well-known POD, the spectral POD (S-POD) recently introduced by Sieber et al. (2016). Benefiting from the bandpass behavior of the S-POD, the goal is to account for the long-range coherence of the most energetic coherent structures existing in the boundary layer to first extract them and then to allow for the study of their interactions with the near canopy turbulence. The remainder of the paper is organized as follows: the experimental setup, the main characteristics of the flow and the POD procedure are presented in Sect. 2. The results obtained from the proposed POD analysis are presented and discussed in Sect. 3. The last section is devoted to the conclusions.

\section{Methods}

In this section, a description of the experimental apparatus and procedures and a presentation of the characteristics of the generated boundary layer are provided. The obstacle height $h$ has been chosen large enough to ensure both large Reynolds numbers $h^{+}=h u_{\tau} / v=1430$ and $z_{0}^{+}=z_{0} u_{\tau} / v=160$ (where $v$ is the kinematic velocity) guaranteeing that the flow is in a fully rough regime, and a satisfying spatial resolution of the PIV measurements. The trade-off for obtaining a ratio $\delta / h \simeq 20$ is the rather limited streamwise extent of the PIV field of view when compared to the typical integral length scales of the boundary layer. It is indeed limited to a fraction of $\delta$, which constitutes another motivation for the development of a new scale-decomposition method. The obtained scaling ratios $\delta / h$ and $z_{0} / h$ (see Table 1) are in the range of those discussed in the introduction, ensuring the investigated flow presents the same characteristics as the targeted flow configurations (Cheng et al. 2007; Grimmond and Oke 1999).

In the following, $x, y$ and $z$ denote the streamwise, spanwise and wall-normal directions, respectively (Fig. 1), and $u, v, w$ the streamwise, spanwise and wall-normal velocity components, respectively. Using the Reynolds decomposition, each instantaneous quantity $u$ can be decomposed as $u=\bar{u}+u^{\prime}$ where $\bar{u}$ denotes the ensemble average (equivalent to a time-average) of $u$ and $u^{\prime}$ its fluctuating part. The spatial average over the streamwise extent of the SPIV measurement region of $u$ is denoted $\langle u\rangle$. The variance of any quantity $u$ obtained via combined time and spatial is denoted $\sigma_{u}^{2}$.

\subsection{Experimental setup}

The present experiments were performed in the atmospheric boundary layer wind tunnel of the LHEEA lab, Nantes, France. It has working section dimensions of $24 \times 2 \times 2 \mathrm{~m}^{3}$ and a freestream velocity range of $3-10 \mathrm{~ms}^{-1}$. The simulation at the scale 1:200 of a suburban-type atmospheric boundary layer developing over an idealized canopy model was achieved using three wall-normal, tapered spires of height of $800 \mathrm{~mm}$ and width of $134 \mathrm{~mm}$ at their base, a 200-mmhigh solid fence across the working section located $0.75 \mathrm{~m}$ 
Fig. 1 Wind tunnel layout (adapted from Blackman and Perret 2016)

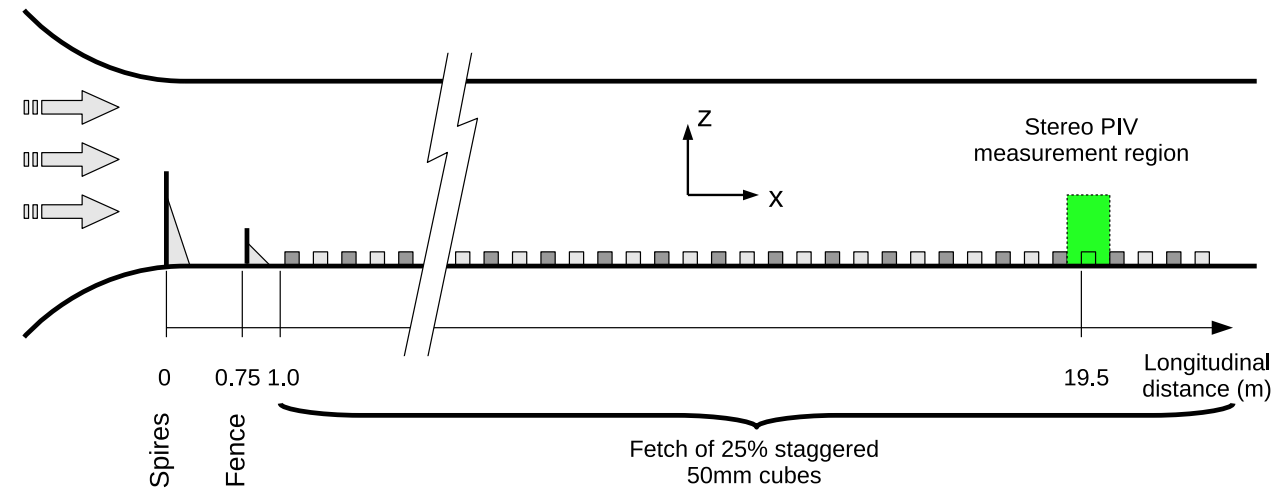

downstream of the inlet, followed by a $22-\mathrm{m}$ stretch of staggered cube roughness elements with a plan area density of $\lambda_{\mathrm{p}}=25 \%$ (Rivet et al. 2012). The cube height was $h=50 \mathrm{~mm}$. The measurements were performed with a freestream velocity, $U_{\mathrm{e}}$, of $5.77 \mathrm{~ms}^{-1}$, at a streamwise location of $19.5 \mathrm{~m}$ after the end of the contraction. Characteristics of the generated boundary layer are described in the following section. For a complete description and characterization of the flow, the reader is referred to the work of Perret et al. (2019).

To fully characterize the boundary layer, single hotwire measurements were performed along the wall-normal direction throughout the whole boundary layer (Perret et al. 2019). For that purpose, only one location relative to a cube was considered as spatial heterogeneities of the flow induced by the presence of the cubes are confined to the lower region of the flow below $z / h \simeq 2-3$. Blackman et al. (2017) and Basley et al. (2018) have indeed shown that second-order statistics and terms in the turbulent kinetic energy budget were uniform in the horizontal plane above that height. A DANTEC 55P11 probe (with a wire length of $1.25 \mathrm{~mm}$ corresponding to $l^{+}=l u_{\tau} / v=30$ to 56 wall units) with a DISA $55 \mathrm{M} 01$ electronics was used with an overheat ratio set to 1.8 , combined with an eighth-order anti-aliasing linear phase elliptic low-pass filter used before signal digitization. Acquisitions were performed during a period of approximately $24,000 \delta / U_{\mathrm{e}}$ using a sampling frequency of $10 \mathrm{kHz}$. A total of 39 logarithmically distributed points were sampled. The temperature correction procedure proposed by Hultmark and Smits (2010) was employed and included in a calibration procedure based on King's law. The probe was calibrated in the freestream flow at the beginning of each half of the profile. Both the form drag and the displacement height $d$ were estimated using the method proposed by Cheng et al. (2007). Distribution of the wall pressure on the back and front faces of a cube obstacle was measured. A cube was manufactured with 36 pressure ports on a wall-normal face to measure the pressure difference between the wall-pressure on the cube and the static pressure in the freestream. The cube was rotated to access the pressure distribution on both the upstream and downstream faces successively. The friction velocity $u_{\tau}$ has been obtained from the calculation of the form drag via the direct integration of the pressure forces onto the instrumented cube. As shown below, the flow being in fully rough regime, the contribution of the viscous forces to the total drag has been neglected (Cheng et al. 2007; Jimenez 2004). Jackson (1981) interpreted the displacement height $d$ as the height at which the drag on the roughness elements is exerted. Therefore, using the same set of mean pressure measurements, the displacement height $d$ was inferred from the calculation of the moment of the pressure forces about the base of the cube. The roughness length $z_{0}$ has been obtained by fitting the measured mean velocity profile with the logarithmic law of Eq. 1.

Stereoscopic PIV measurements were conducted in a wall-normal plane aligned with the main flow, in the center of the test section (Fig. 1). Details of the exact location of the measurement plane are shown in Fig. 2. Two $2048 \times 2048$ pixels $^{2}$ 12-bit camera equipped with Nikon $105 \mathrm{~mm} \mathrm{AF}$ Micro objective lenses (at aperture $f_{\#}=2.8$ ) were employed in a stereoscopic configuration. The Scheimpflug condition was satisfied by rotating the image plane with respect to the lens plane via the use of dedicated remote-controlled mounts. The cameras were mounted outside the test section, on each side of the light sheet in a symmetric configuration, each camera axis forming an angle of $48^{\circ}$ with the $x-z$ plane. The final spatial resolution is $1.72 \mathrm{~mm}$ ( 49 wall units) and $2.20 \mathrm{~mm}$ (63 wall units) in the streamwise and wallnormal directions, respectively. A Litron $200 \mathrm{~mJ} \mathrm{Nd-YAG}$ laser, located under the wind tunnel floor, was employed to illuminate the region of interest through a glass window. The laser sheet thickness was estimated as being $3 \mathrm{~mm}$. The time delay between two images for PIV processing was set to $d t=500 \mu \mathrm{m}$, ensuring an average displacement of 13 pixels over the entire region of interest. The flow was seeded with glycol/water droplets (typical size $1 \mu \mathrm{m}$ ) using a fog generator. Both the synchronization between the laser and the cameras and the calculation of the PIV velocity vector fields were performed using the DANTEC FlowDynamics software. A set of $N=4000$ velocity fields was recorded with 
Fig. 2 Configuration of measurement. The dotted line shows the location of the PIV measurement plane in a the $x-z$ plane and $\mathbf{b}$ the $y-z$ plane. The red dashed line and the red cross show the location of the additional hot-wire measurements performed for the flow characterization (a)

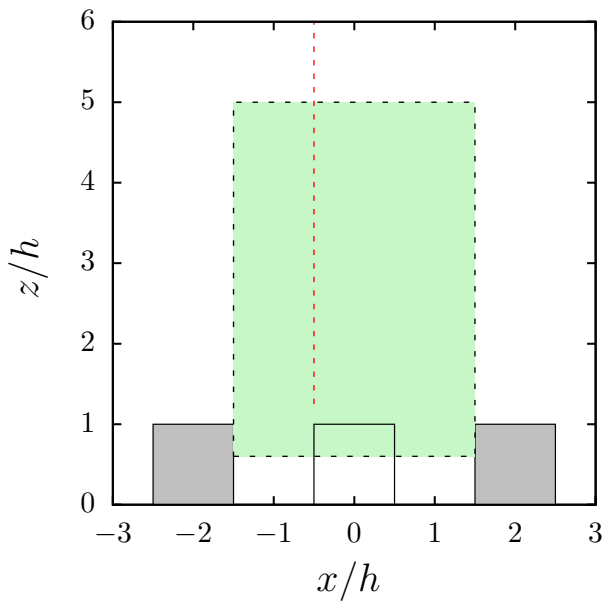

(b)

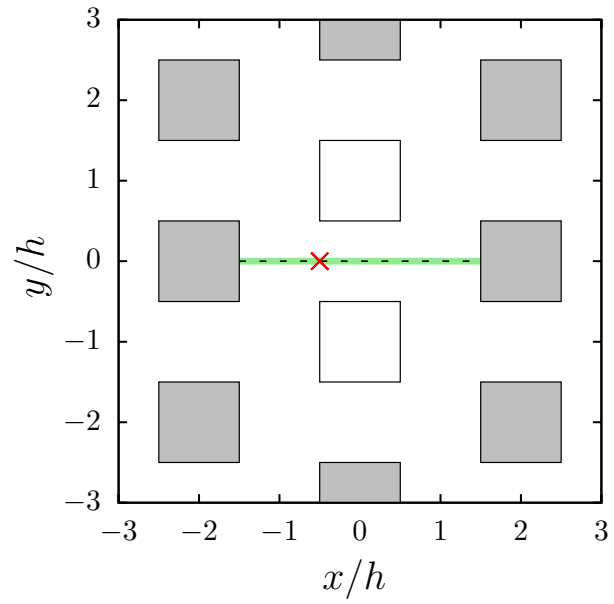

a sampling frequency of $f_{\mathrm{s}}=5 \mathrm{~Hz}$ to enable the computation of the main flow statistics and investigate its large-scale organization. A FFT-based 2D-PIV algorithm with sub-pixel refinement was employed. Iterative cross-correlation analysis was performed with an initial window size of $128 \times 128$ pixels and with $32 \times 32$ final interrogation windows, using an overlap of $50 \%$. Spurious vectors were detected by an automatic validation procedure whereby the signal to noise ratio of the correlation peak had to exceed a minimum value, and the vector amplitude had to be within a certain range of the local median to be considered as valid. Once spurious vectors had been detected, they were replaced by vectors resulting from a linear interpolation in each direction from the surrounding $3 \times 3$ set of vectors. A pinhole model was employed to reconstruct the three-component vector fields from the two-component vector fields from each cameras (Soloff et al. 1997; Calluaud and David 2004).

\subsection{Boundary layer characteristics}

The main characteristics of the investigated boundary layer are summarized in Table 1. As mentioned earlier, the ratio $\delta / h$ and $z_{0} / h$ are in the range of those expected for the type of targeted urban terrain. Moreover, both the Reynolds number based on the obstacle height $h^{+}$and that based on the 99\% boundary layer thickness $\delta^{+}=\delta u_{\tau} / v$ are large enough to ensure that the flow is in the fully rough regime (Jimenez 2004) and that the present boundary layer can be qualified as a high-Reynolds number one. This is confirmed by the value of $z_{0}^{+}$, largely above the critical value of 0.5 reported by Snyder and Castro (2002). The value of $d / h$ and $z_{0} / h$ are in good agreement with those reported in the literature (Castro et al. 2006; Cheng et al. 2007; Grimmond and Oke 1999), confirming the suitability of the present setup for reproducing the aerodynamical characteristics of the targeted flow.

Wall-normal profiles of the mean streamwise velocity obtained from both PIV and hot-wire measurements at a single streamwise location (Fig. 2) are presented in Fig. 3a and show good agreement between each other. Moreover, the profile of the mean streamwise velocity component exhibits a well-developed logarithmic region. An excellent match with Eq. 1 is obtained, confirming the high-Reynolds number character of the flow. The profiles of the streamwise velocity variance (Fig. 3a) show also an excellent agreement except near the top of the obstacles. The discrepancy between the two measurement techniques in the lowest part of the flow $\left((z-d) / z_{0} \leq 9\right.$ or $\left.z / h \leq 1.5\right)$ is due to the slightly lower spatial resolution of the PIV measurements which induces a low-pass filter effect on the velocity fluctuations (Herpin et al. 2018).

Pre-multiplied spectrum of the streamwise velocity is shown as a function of wall-normal distance and Strouhal number $f \delta / \bar{u}(z)$ in Fig. 3b. In high-Reynolds number turbulent boundary layers over smooth wall, such pre-multiplied spectra exhibit two distinct maxima, one in the outer region attributed to the predominance of the VLSMs, for streamwise wavelength $\lambda / \delta \approx 6$, and one in the near-wall region associated with smaller scale turbulence, for $\lambda^{+} \approx 1000$ (Mathis et al. 2009). At the Reynolds number of the present experiment, these wavelengths would correspond to Strouhal numbers $f \delta / \bar{u}(z)$ of 0.17 and 32 , respectively. In the present case, no such distinction can be made. In the outer
Table 1 Main characteristics of the boundary layer

\begin{tabular}{llllllll}
\hline$\lambda_{\mathrm{p}}(\%)$ & $U_{\mathrm{e}}\left(\mathrm{ms}^{-1}\right)$ & $u_{\tau} / U_{\mathrm{e}}$ & $\delta / h$ & $h^{+}$ & $\delta^{+}$ & $d / h$ & $z_{0} / h$ \\
\hline 25 & 5.77 & 0.074 & 22.7 & 1430 & 32,400 & 0.59 & 0.11 \\
\hline
\end{tabular}


(a)

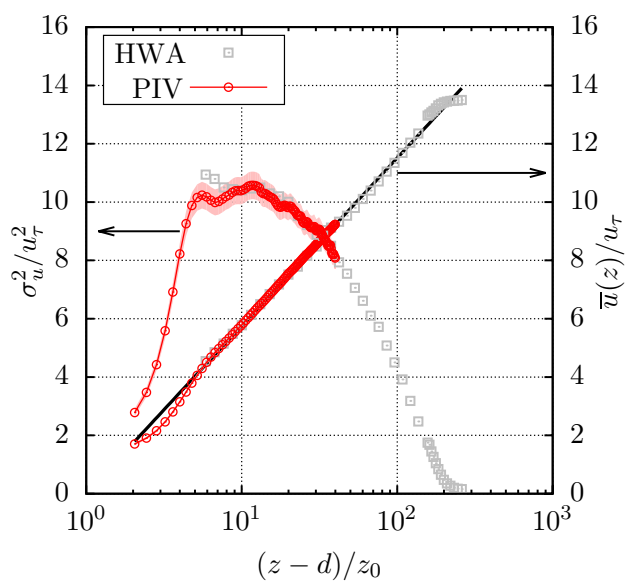

Fig. 3 Statistics of the streamwise velocity $u$. a Profiles of mean and variance obtained by (red open circles) PIV and (gray open squares) hot-wire anemometry at the same streamwise location ( $1 h$ downstream the upstream cube). Light red-shaded areas represent the statistical errors of the PIV statistics with a $95 \%$ confidence level. Statistical errors for the HWA measurements being below $1 \%$ are not

region, the ridge of the pre-multiplied spectra corresponds to $f \delta / \bar{u}(z) \approx 0.3$ while at the location closest to the canopy, the maximum of energy is obtained for $f \delta / \bar{u}(z) \approx 2$, which is an order of magnitude smaller than in the smooth-wall case. It is likely that the presence of the cubical obstacles induces energetic structures with typical frequencies smaller than that of the near-smooth-wall turbulence, in a range closer to those attributed to the large-scale structures developing in the logarithmic and outer region. The absence of two distinct maxima in the spectral domain prevents us from separating the larger scale contribution from the near-canopy turbulence using a simple low-pass filter on the HWA measurements as employed by Mathis et al. (2009). Finally, it must be noted that the Nyquist frequency $f_{\mathrm{s}} / 2$ of the present PIV system is above that of the most energetic scales as shown by the solid green line in Fig. 3b. As demonstrated in the next section, this will allow us to separate the most energetic (large) scales populating the boundary layer from turbulent scales developing in the near-canopy region.

\subsection{POD-based method for scale separation}

In this section, the method of analysis of the flow field which also serves as a scale separation filter is presented. The now well-known snapshot POD is first introduced. As also discussed in Sect. 3, when applied to data measured in a spatial domain of limited spatial extent with a low temporal resolution, this method is not able to correctly capture the presence and the organization of the large-scale energetic structures populating the boundary layer. As evidenced (b)

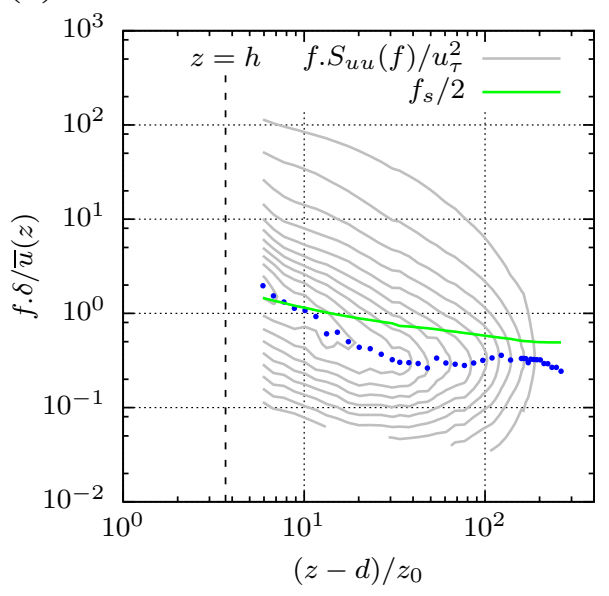

shown. The solid black line shows the logarithmic law of Eq. (1). b Pre-multiplied spectra $f . S_{u u}(f) / u_{\tau}^{2}$ (gray contours, shown with an increment of 0.08 , from 0.08 to 0.88 ). Blue filled circles indicate the local maxima of the pre-multiplied spectra; the thick solid green line shows the Nyquist frequency $f_{\mathrm{s}} / 2$ associated with the low-rate PIV system

by Towne et al. (2018) and demonstrated by Mendez et al. (2018), the classical POD, while allowing for the best convergence, is not able to properly account for the dynamics of different coherent structures of similar energy, therefore, producing spectral mixing of different phenomena over different modes. Moreover, the PIV is by nature a method that samples the flow producing discrete time information without the possibility of implementing an analog low-pass filter prior digitization. Therefore, from a temporal point of view, when the acquisition frequency of the PIV system is low compared to the entire spectral content of the flow, the classical spectral aliasing phenomenon (in the temporal domain) is expected to occur. In the present case, given the low PIV sampling frequency of $5 \mathrm{~Hz}$ (and even if it is higher than the typical frequency of the most energetic structures in the boundary layer, see Fig. 3), only the first POD mode appears to bear the footprint of the low-frequency largescale energetic LSMs of the boundary layer. This is shown in Fig. 4 where the auto-correlation function of the first temporal POD coefficients $a_{i}(t)$ (see Sect. 2.3.1 for a complete definition) computed from the SPIV data is reported. As can be seen, only the auto-correlation of the first temporal mode is well resolved. The latter is well approximated by an exponential decaying function of the form $\mathrm{e}^{(-\tau / T)}$ with $T$ the integral timescale which, when Taylor's hypothesis is used, can be related to a characteristic length scale $L_{x} \approx 24 h$ or $1.05 \delta$, confirming that the first mode is associated with largescale motion. Yet, the spatio-temporal dynamics of these LSMs is richer than what can be represented by one single mode and is, therefore, not correctly captured. To overcome 


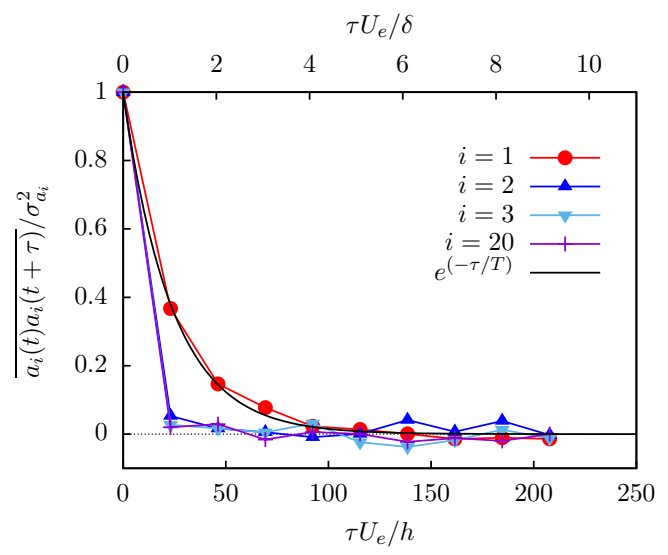

Fig. 4 Normalized temporal auto-correlation function of the classical snapshot POD temporal modes $a_{i}(t)$ as defined in Eq. 3, for $i=1,2,3$ and 20

this limitation, a modification of the snapshot POD is proposed based on the recent results of Sieber et al. (2016). In the new method, the POD kernel is computed so as to take into account the long-term temporal coherence of the largest scales. This modified POD approach enables the de-aliasing of the first POD modes which will be shown to bear all the characteristics of the LSMs identified in the current literature. Such an approach has been formalized by Sieber et al. (2016). The authors refer to this POD method as spectral POD as they showed that it behaves as a band-pass filter in the frequency domain. It must be noted that this variant of POD is different from the space-time formulation of POD originally proposed by Lumley (1967), which involves the decomposition of the cross-spectral density tensor and has hence later been referred to as spectral POD as well.

\subsubsection{Classical snapshot POD}

Lumley (1967) first proposed the POD technique to identify the coherent structures in turbulent flows. When limited to space only, it consists in extracting from the flow the structure $\phi(X)$ with the largest mean-square projection onto the fluctuating velocity field $u^{\prime}(\boldsymbol{X}, t)$ over the spatial domain of interest. This maximization problem leads to the solving of an integral problem of eigenvalues. It allows for the decomposition of the fluctuating velocity field $u^{\prime}$ into a set of temporal and spatial orthogonal modes $a_{i}(t)$ and $\phi_{i}(\boldsymbol{X})$ such that, in the most general case,

$u^{\prime}(\boldsymbol{X}, t)=\sum_{i=1}^{\infty} a_{i}(t) \phi_{i}(\boldsymbol{X})$

In the present work, with the PIV data being well resolved in space but with a limited number of time samples, the snapshot POD proposed by Sirovich (1987) has been employed.
The number of PIV snapshots (or time steps) is denoted $N$. In that case, the integral eigenvalue problem to solve is

$\boldsymbol{R} \boldsymbol{a}_{i}=\lambda_{i} \boldsymbol{a}_{i}$,

where $\lambda_{i}$ is the $i$ th eigenvalue which represents the turbulent energy contained in the $i$ th mode. The matrix $R \in \mathcal{R}^{N \times N}$ defines the temporal cross-correlation matrix (or kernel) whose elements $(i, j)$ are computed as

$R_{i j}=\frac{1}{N} \int_{V} u^{\prime}\left(\boldsymbol{X}, t_{i}\right) u^{\prime}\left(\boldsymbol{X}, t_{j}\right) \mathrm{d} V$,

where $V$ is the spatial domain over which the measurements have been performed. The temporal eigenvectors $\boldsymbol{a}_{i}$ are uncorrelated in time:

$\boldsymbol{a}_{i} \boldsymbol{a}_{j}=\overline{a_{i}(t) a_{j}(t)}=\frac{1}{N} \sum_{k=1}^{N} a_{i}\left(t_{k}\right) a_{j}\left(t_{k}\right)=\lambda_{i} \delta_{i j}$,

while the spatial modes are orthonormal and computed by

$\phi_{i}(\boldsymbol{X})=\frac{1}{\lambda_{i}} \boldsymbol{a}_{i} \boldsymbol{u}^{\prime}(\boldsymbol{X})$.

\subsubsection{Spectral POD}

Sieber et al. (2016) have recently introduced a modification of the temporal correlation matrix $\boldsymbol{R}$ originally used in the conventional snapshot POD to include an additional temporal constraint that enables a clear separation of phenomena that occur at multiple frequencies and energies. They proposed to apply a low-pass filter $\boldsymbol{g}$ along the diagonals of $\boldsymbol{R}$ to augment its diagonal similarity. A new temporal correlation matrix $S$ is obtained by first integrating the velocity product over the spatial domain $V$ and then by applying the filter temporal function $g$ :

$S_{i j}=\frac{1}{N} \sum_{k=-N_{\mathrm{f}}}^{N_{\mathrm{f}}} \int_{V} g_{k} u^{\prime}\left(\boldsymbol{X}, t_{i+k}\right) u^{\prime}\left(\boldsymbol{X}, t_{j+k}\right) \mathrm{d} V$.

The function $\boldsymbol{g}$ is a symmetric finite impulse response filter of width $2 N_{\mathrm{f}}+1$. In the simplest case, $\boldsymbol{g}$ would be a box filter of value $1 /\left(2 N_{\mathrm{f}}+1\right)$ for $k \in\left[-N_{\mathrm{f}} ; N_{\mathrm{f}}\right]$, and zero elsewhere. As discussed by Sieber et al. (2016), a Gaussian filter can be employed to obtain a smooth response in time and frequency domaind. The eigenvalue problem to solve becomes

$\boldsymbol{S} \boldsymbol{b}_{i}=\mu_{i} \boldsymbol{b}_{i} ; \quad \mu_{1} \geq \mu_{2} \geq \cdots \geq \mu_{N}$,

where $\mu_{i}$ is the eigenvalue of the temporal S-POD mode $\boldsymbol{b}_{i}$. The modes $\boldsymbol{b}$ are orthogonal (i.e. uncorrelated in time):

$\boldsymbol{b}_{i} \boldsymbol{b}_{j}=\mu_{i} \delta_{i j}$. 
In contrast to Sieber et al. (2016), the temporal S-POD modes are now projected onto time-delayed fluctuating velocity fields to obtain spatio-temporal S-POD modes:

$\boldsymbol{\Psi}_{i}\left(\boldsymbol{X}, \tau_{k}\right)=\frac{1}{\mu_{i}} \sum_{l=1+k}^{N-k} b_{i}\left(t_{l}\right) u^{\prime}\left(\boldsymbol{X}, t_{l+k}\right)$.

The index $k \in\left[-N_{\mathrm{f}} ; N_{\mathrm{f}}\right]$ corresponds to a time delay $\tau_{k}=k / f_{\mathrm{s}} \in\left[-T_{\mathrm{f}} ; T_{\mathrm{f}}\right]$, where $T_{\mathrm{f}}=N_{\mathrm{f}} / f_{\mathrm{s}}$ is the half-width of the filter function $g$. For the sake of brevity, the modes $\boldsymbol{\Psi}_{i}$ are referred to as spatial modes in the following. These spatial S-POD modes are orthonormal if the following inner product is employed:

$$
\sum_{k=-N_{\mathrm{f}}}^{N_{\mathrm{f}}} \frac{1}{V} \int_{V} \boldsymbol{\Psi}_{i}\left(\boldsymbol{X}, \tau_{k}\right) \boldsymbol{\Psi}_{j}\left(\boldsymbol{X}, \tau_{k}\right) \mathrm{d} V=\delta_{i j}
$$

It must be noted here that the present definition of the spatial S-POD modes is different from that proposed by Sieber et al. (2016). These authors indeed originally defined these modes as being dependent on space only by not including the integration over the temporal span of the low-pass filter in Eq. 12. As mentioned by Sieber et al. (2016), the modes are not orthogonal if defined in this manner. Therefore, by including their dependence on the time delay $\tau$, the present modified definition of the modes $\boldsymbol{\Psi}$ leads to orthonormal modes, in agreement with the original POD approach. The original velocity field can be reconstructed by considering the spatio-temporal modes at zero time as

$u(\boldsymbol{X}, t)=\bar{u}(\boldsymbol{X})+\sum_{i=1}^{N} b_{i}(t) \boldsymbol{\Psi}_{i}(\boldsymbol{X}, \tau=0)$.

The definition of the spatio-temporal modes proposed here does not alter any other property of the spectral POD as designed by Sieber et al. (2016). As shown by Eq. 8, the S-POD acts as a band-pass filter centered around the dominant frequency, whose half-width $T_{\mathrm{f}}$ is controlled by the characteristics of the low-pass filter $g(\tau)$. Given the value of $N_{\mathrm{f}}$, two asymptotic cases exist: if $N_{\mathrm{f}}=0$, the S-POD is equivalent to the conventional POD, while for $N_{\mathrm{f}}=+\infty$, it corresponds to the discrete Fourier transform. To benefit from the band-pass filtering effect of the S-POD, Sieber et al. (2016) advise to choose a value of $N_{\mathrm{f}}$ so that the filter half-width $T_{\mathrm{f}}$ corresponds to two to three times the characteristic timescale of the phenomena of interest. In the present study, a Gaussian filter is used for $g$. The most energetic frequencies in the outer region are of the order $f \delta / \bar{u}(z) \approx 0.3$ (Fig. 3b). In the upper region of the SPIV field of view, this corresponds to frequencies of approximately $1 \mathrm{~Hz}$. Given the acquisition frequency of the PIV system of $f_{\mathrm{s}}=5 \mathrm{~Hz}$, $N_{\mathrm{f}}$ should be equal to 15 to ensure that the filter width spans three times this characteristic timescale. In the following, it is shown that a value of $N_{\mathrm{f}}=16$ leads to satisfactory results in terms of large-scale extraction.

\section{Results}

Both the conventional (i.e. $N_{\mathrm{f}}=0$ ) and spectral snapshot POD detailed previously are now employed. Only the streamwise velocity component $u$ is considered. The spatial eigenvectors $\phi$ and $\boldsymbol{\Psi}$ are function of the streamwise $x$ and wall-normal $z$ coordinates. Using S-POD, the fluctuations of the streamwise velocity component $u$ are then decomposed into $u^{\prime}=u_{\mathrm{L}}^{\prime}+u_{\mathrm{S}}^{\prime}$, where $u_{\mathrm{S}}^{\prime}$ is the small-scale component of $u^{\prime}$ and $u_{\mathrm{L}}^{\prime}$ the large-scale component defined as

$u_{\mathrm{L}}^{\prime}(x, z)=\sum_{i=1}^{N_{\mathrm{POD}}} b_{i}(t) \Psi_{i}(x, z, \tau=0)$,

where $N_{\text {POD }}$ is the number of POD modes selected to define the large-scale component.

\subsection{S-POD analysis}

In the following, most of the results correspond to the case $N_{\mathrm{f}}=16$, the value for which the low-pass filter width $T_{\mathrm{f}}$ is equal to three times the characteristic timescale of the phenomena of interest. While a systematic study of the effect of $N_{\mathrm{f}}$ on the results has been performed, it is not reported here for conciseness. As described by Sieber et al. (2016) in detail, the influence of varying $N_{\mathrm{f}}$ can be directly seen on the frequency content of the temporal S-POD coefficients $b_{i}(t)$. The S-POD acts as a band-pass filter applied to the $b_{i}(t)$, the width (in the frequency domain) of which varying from $+\infty\left(N_{\mathrm{f}}=0\right.$, equivalent to the classical POD) to the narrowest possible one, dictated by the PIV sampling of the flow $\left(N_{\mathrm{f}}=+\infty\right.$, equivalent to the Fourier transform). The frequency around which the band-pass filter is applied is intrinsically selected by the S-POD, based on the energetic optimality criterion underpinning POD. The choice of $N_{\mathrm{f}}$ results in a compromise between the fastest energetic convergence (POD) and the best frequency resolution (Fourier decomposition). This is effect is readily visible on the eigenspectrum.

As mentioned earlier, given the low acquisition frequency $f_{\mathrm{s}}$ and the limited field of view of the employed SPIV system, a major drawback of the conventional snapshot POD (i.e. $N_{\mathrm{f}}=0$ ) is the spectral mixing of different coherent structures over different modes and the aliasing of the low-order modes by the smaller scales. These most energetic modes which should correspond to slow-varying large scales of the present boundary layer do not present 
Fig. 5 Phase portrait of the first two normalized temporal S-POD coefficients of $u$ for a $N_{\mathrm{f}}=0\left(\tilde{a}_{i}=a_{i} / \sqrt{\lambda_{i}}\right)$ and $\mathbf{b}$ $N_{\mathrm{f}}=16\left(\tilde{b}_{i}=b_{i} / \sqrt{\mu_{i}}\right)$
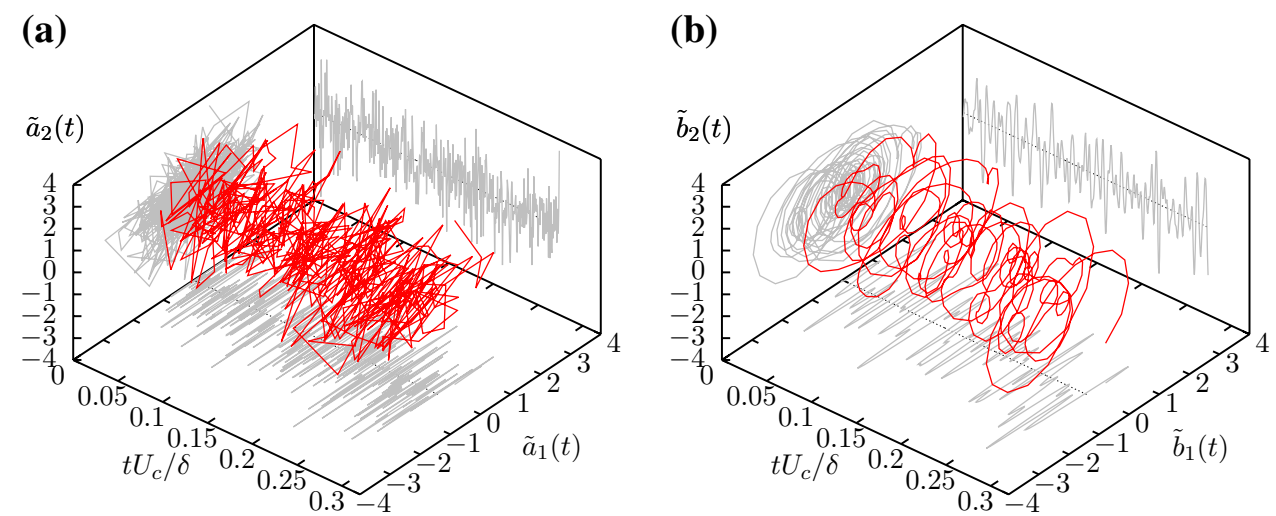

(a)

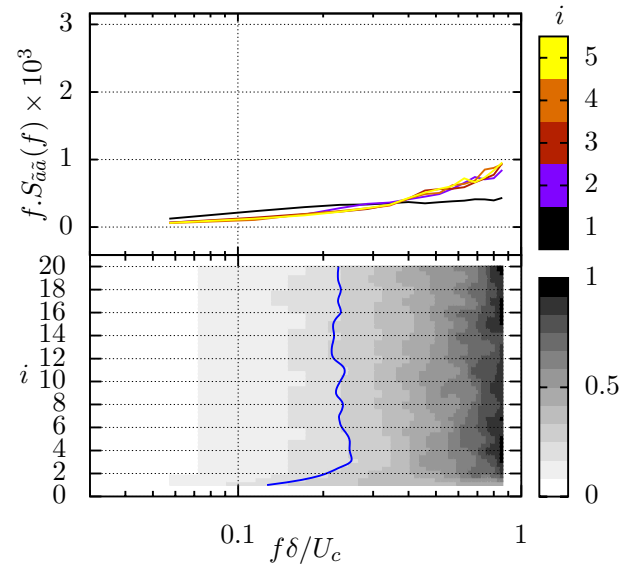

(b)

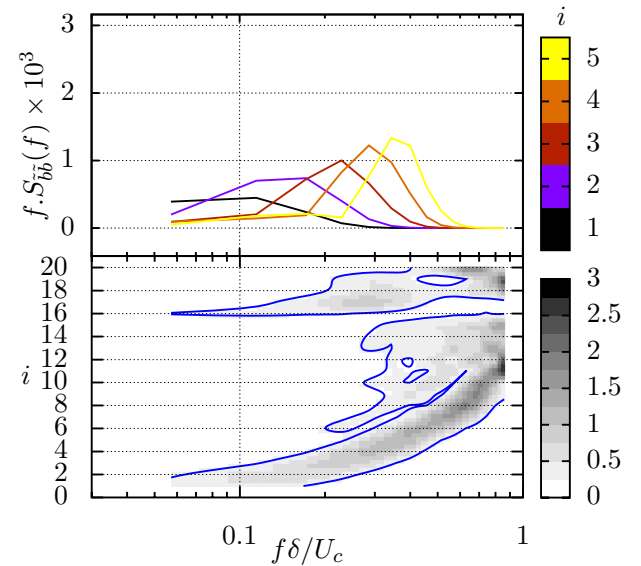

$f \delta / U_{c}$
Fig. 6 Pre-multiplied spectra of temporal S-POD normalized coefficients for $\mathbf{a} N_{\mathrm{f}}=0$ and b $N_{\mathrm{f}}=16$. The upper part of the plot shows pre-multiplied spectra of the first five temporal S-POD coefficients (colored by the mode order $i$ ), the bottom part the pre-multiplied spectra contours in shades of gray for the first 20 modes. The solid blue line shows the 0.25 contour a smooth time evolution with typical frequencies below $f_{\mathrm{s}} / 2$. This is illustrated in Fig. 5a where the phase portrait of the first two normalized temporal coefficients $\tilde{a}_{1}$ and $\tilde{a}_{2}$ and their time traces are shown as a function of time. No apparent continuity in time between two consecutive samples is visible. Therefore, even if the first S-POD mode obtained with $N_{\mathrm{f}}=0$ is able to capture the long-term time correlation of the largest scales (Fig. 4), the phase relationship with subsequent modes is lost. On the contrary, when the temporal coherence of the most energetic scales is taken into account by setting $N_{\mathrm{f}}=16$, the band-pass filtering effect of S-POD allows for the proper time resolution of the first S-POD modes (Fig. 5b). This is confirmed by the computation of the pre-multiplied energy spectra of the normalized temporal coefficients $\tilde{a}_{i}$ and $\tilde{b}_{i}$, for $i \in[1: 20]$ (Fig. 6). When $N_{\mathrm{f}}=0$, all the temporal modes show an accumulation of energy at high frequency without a clear well-defined spectral content, which is typical of the aliasing phenomena (Fig. 6a). Conversely, setting $N_{\mathrm{f}}=16$ leads to a clear band-pass filtering of the energy content (Fig. 6b). The energy of the first S-POD modes is clearly contained in a limited frequency band, the corresponding most energetic frequency increasing with the (a)

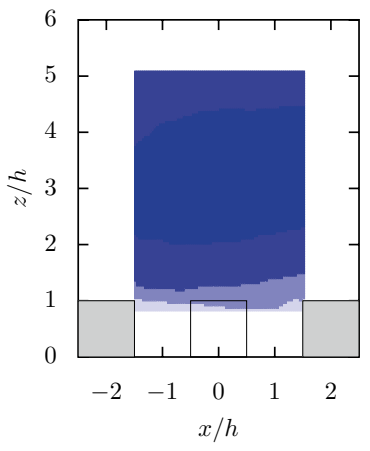

(b)

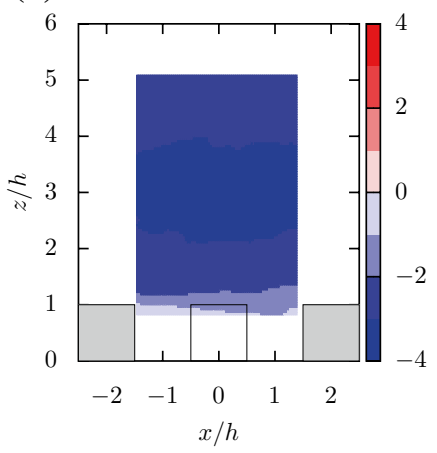

Fig. 7 First spatial S-POD modes $\Psi_{i}(x, z, \tau=0)$ of $u$ with a $N_{\mathrm{f}}=0$ and $\mathbf{b} N_{\mathrm{f}}=16$

order of the mode $i$. When $i>5$, a secondary peak at lower frequency appears, which can be attributed again to aliasing. Therefore, with the present SPIV setup, the use of S-POD with the proper filter width (set in accordance with the characteristic frequencies of the most energetic structures existing in the outer of the boundary layer) allows for the de-aliasing of the largest scales (here limited to $i \leq 5$ ). 
When analyzing the spatial structures of the first S-POD mode, the influence of the filter width is not that obvious. The first eigenvectors of the POD and S-POD (for $\tau=0$ ) are shown in Fig. 7. In both cases, the first spatial modes obtained are very similar and correspond to the occurrence of high- (or low-)speed events in the flow spanning the whole region $z>1.5 h$.

However, using S-POD with $N_{\mathrm{f}} \neq 0$ leads to spatio-temporal modes $\Psi(x, z, \tau)$ which bear the imprint of the long-term coherence of the large most energetic scales of the flow. The first five S-POD modes obtained with $N_{\mathrm{f}}=16$ are shown in Fig. 8. To represent the spatio-temporal structure of the S-POD modes, Taylor's approximation is here used to transform the time delay $\tau$ in a pseudo-streamwise spatial coordinate with an advection velocity $U_{\mathrm{c}}$ corresponding to the average mean velocity across the measurement region. Estimating the actual convection velocity of the large-scale coherent structures, which is known to be a function of the wall-normal distance and the considered scale (Dennis and Nickels 2008), is out of the scope of the present study and not further investigated. Even if Taylor's approximation is known to have limitations in the region of spatial heterogeneity and high level of turbulence intensity, it is employed here for the sake of displaying the

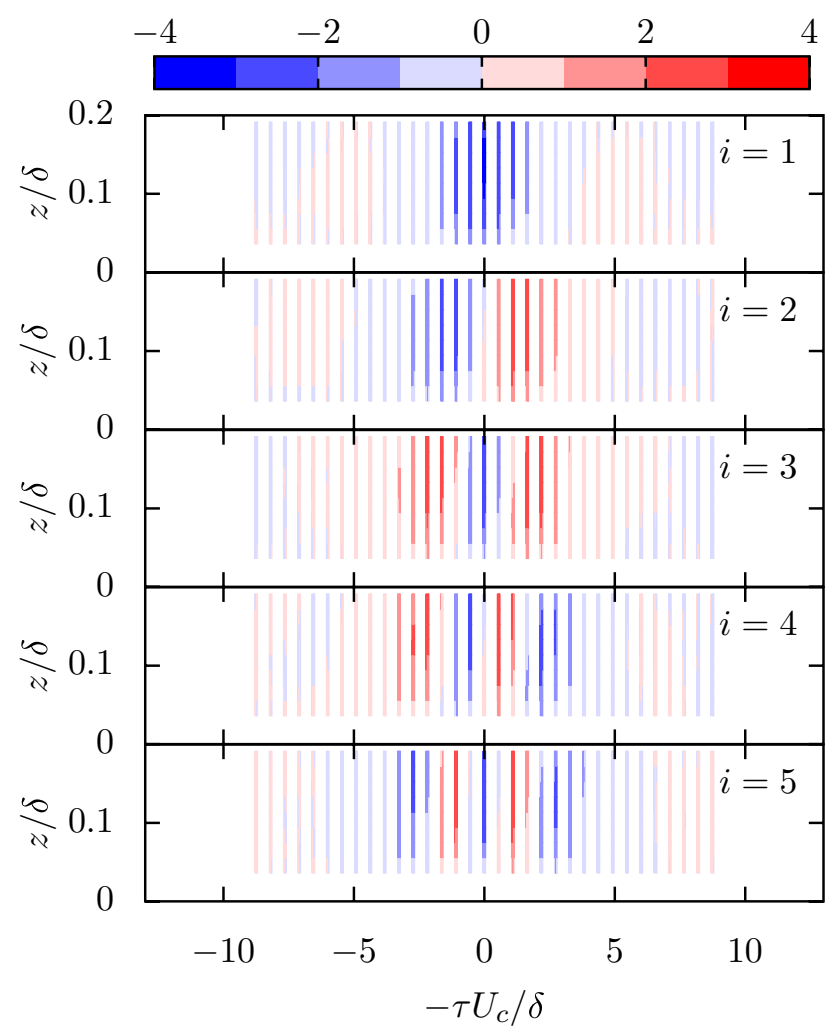

Fig. 8 First five spatial S-POD modes $\Psi(x, z, \tau)$ of $u$ with $N_{\mathrm{f}}=16$. The size of each colored strip corresponds to that of the SPIV field of view spatio-temporal structure of the S-POD modes $\Psi(x, z, \tau)$. The size of each colored strip in Fig. 8 corresponds to that of the SPIV field of view. Each mode depicts an oscillatory behavior along the temporal direction, the typical scale of which being of the order of several boundary layer height $\delta$. This characteristic is in perfect agreement with that of the VLSMs evidenced in boundary layer flows using more conventional techniques (Guala et al. 2011; Hutchins and Marusic 2007). The higher the mode order, the smaller its time period, in agreement with the fact that higher order modes usually correspond to smaller scales, in particular when one direction of analysis of the flow corresponds to the direction of advection of the flow (streamwise and time directions here). Limiting the frequency content of the S-POD modes by setting $N_{\mathrm{f}}>0$ has, however, an effect on the energy content of each mode and on the rate of convergence of the POD decomposition in terms of number of modes required to represent a given amount of kinetic energy. The larger the $N_{\mathrm{f}}$, the slower the convergence, the energy content of each mode being constrained by the band-pass effect of the S-POD method (Fig. 9). Using Eq. 14, the large-scale streamwise velocity component $u_{\mathrm{L}}^{\prime}$ corresponding to the contribution of the first $N_{\mathrm{f}} \mathrm{S}$-POD modes can be reconstructed. In the present case, $N_{\mathrm{POD}}$ is set to five, the number of S-POD modes properly resolved in time by the S-POD when using $N_{\mathrm{f}}=16$. The small-scale streamwise velocity component $u_{\mathrm{S}}^{\prime}$ is calculated as $u_{\mathrm{S}}^{\prime}=u^{\prime}-u_{\mathrm{L}}^{\prime}$. An example of the temporal evolution of the instantaneous streamwise velocity component fluctuation, its large- and small-scale components and their contribution to the shear-stress $u^{\prime} w^{\prime}=u_{\mathrm{L}}^{\prime} w^{\prime}+u_{\mathrm{S}}^{\prime} w^{\prime}$ is shown in Fig. 10. Despite the low PIV acquisition frequency, the temporal evolution of $u^{\prime}$ clearly shows the footprint of large-scale coherent structures with typical time period of a few timescale $\delta / U_{\mathrm{c}}$. The time series of $u_{\mathrm{L}}^{\prime}$ exhibits alternation

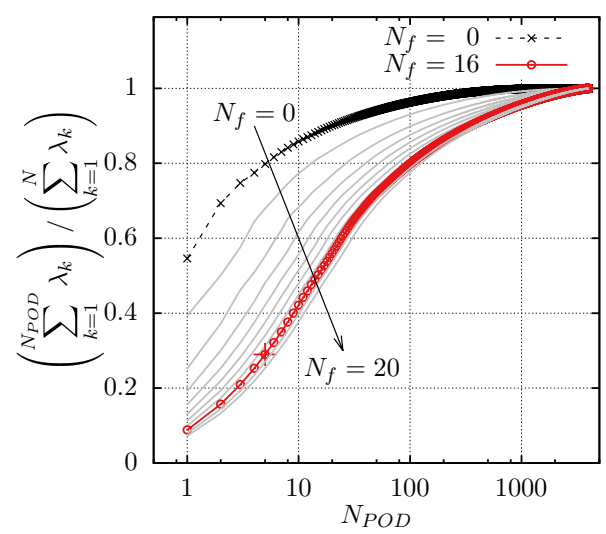

Fig. 9 Energy contribution of the first $N_{\text {POD }}$ S-POD modes of $u$ as a function of the filter size $N_{\mathrm{f}}$. Results are shown for $N_{\mathrm{f}}=0$ to 20 with an increment of 2 . The dashed black line with crosses shows the case $N_{\mathrm{f}}=0$ corresponding to the conventional snapshot POD, the solid red line with open circles shows the $N_{\mathrm{f}}=16$ case. The red plus sign (+) corresponds to $N_{\mathrm{f}}=16, N_{\mathrm{POD}}=5$ 
Fig. 10 Example of the temporal evolution of (from top to bottom) $u^{\prime}, u_{\mathrm{L}}^{\prime}, u_{\mathrm{S}}^{\prime}, u_{\mathrm{L}}^{\prime} w^{\prime}$ and $u_{\mathrm{S}}^{\prime} w^{\prime}$ measured at $f_{\mathrm{s}}=5 \mathrm{~Hz}$ on the centerline of the PIV field of view (all quantities normalized by $u_{\tau}$ ). The horizontal dotted black lines indicate to $z=h$

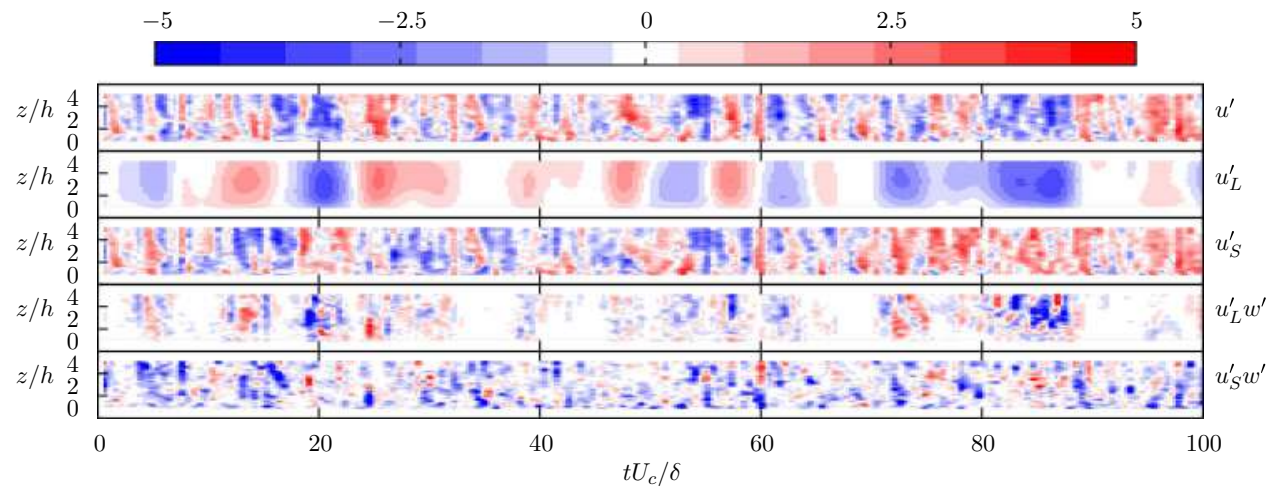

of low- and high-momentum regions with typical time period of the order of at least $10 \delta / U_{\mathrm{c}}$ and spanning the entire wallnormal extent of the PIV field of view. The coherent structures represented by the first $N_{\text {POD }}=5 \mathrm{~S}$-POD modes, therefore, show the typical features of boundary layer VLSMs. The component $u_{\mathrm{S}}^{\prime}$ also shows some smaller timescale organization, less coherent in the wall-normal direction, consistent 1 with the presence of LSMs embedded into the VLSMs. This is supported by the analysis of the instantaneous decomposed shear stress that shows the presence of smaller scale shear stress events, in agreement with the hairpin-vortex packet model (Adrian 2007). The pre-multiplied spectra of $u_{\mathrm{L}}^{\prime}$ are shown in Fig. 11a as a function of the wall-normal coordinate (red contours) and are compared to that obtained with conventional

(a)

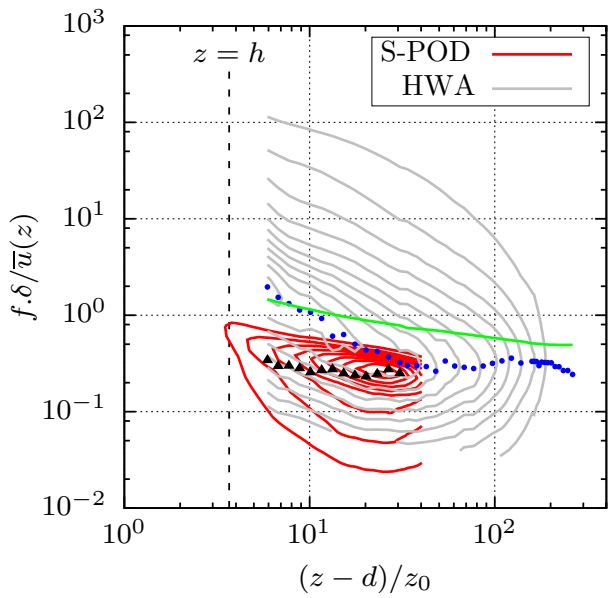

Fig. 11 a Wall-normal evolution of the pre-multiplied spectra $f . S_{u_{\mathrm{L}} u_{\mathrm{L}}}(f) / u_{\tau}^{2}$ of the large-scale streamwise velocity component $u_{\mathrm{L}}^{\prime}$ reconstructed with $N_{\text {POD }}=5\left(N_{\mathrm{f}}=16\right)$ (red solid lines, shown with an increment of 0.04 , from 0.04 to 0.44 ). As in Fig. 3, solid gray lines (shown with an increment of 0.08 , from 0.08 to 0.88 ) correspond to pre-multiplied spectra of $u^{\prime}$ as measured via HWA by Perret et al. (2019), blue filled circles indicate the local maxima of these pre-multiplied spectra; the thick solid green line shows the Nyquist frequency $f_{\mathrm{s}} / 2$ associated to the present low-rate PIV system. Solid black triangles show the most energetic frequency corresponding to
HWA. First, as expected from the above analysis, they do not show any trace of aliasing at high frequency, as if the original velocity field had been properly low-pass filtered. Second, the frequency of the maximum of energy in the upper part of the SPIV measurement region (i.e. $\left.(z-d) / z_{0}>20\right)$ corresponds well to that of the most energetic structures found in the outer region of the boundary layer by Perret et al. (2019). Again, a good agreement of the energy ridge of the pre-multiplied spectra of $u_{\mathrm{L}}^{\prime}$ with the most energetic frequency of the large scales extracted by Perret et al. (2019) is observed across the entire measurement region. Moreover, the present analysis shows that the most energetic (large) scales from the outer layer reach deep into the roughness sublayer, being superimposed onto the smaller scales. The contribution of $u_{\mathrm{L}}^{\prime}$ to the

(b)

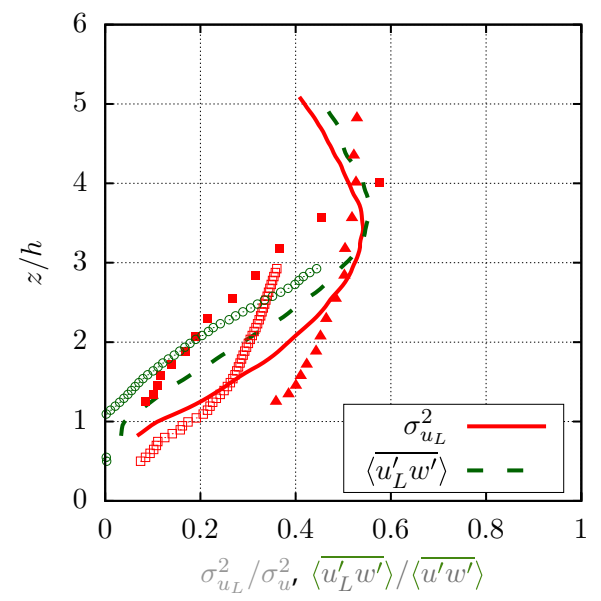

the large scales identified by Perret et al. (2019) via their two-point spectral analysis. b Contribution of the first five S-POD modes to $\sigma_{u}^{2}$ and $\left\langle\overline{\left\langle u^{\prime} w^{\prime}\right.}\right\rangle$. Red solid line: $\sigma_{u}^{2}$ of the present study, green dashed line: $\left\langle\overline{u^{\prime} w^{\prime}}\right\rangle$ of the present study. Red symbol: results for $\sigma_{u}^{2}$. Filled and open squares correspond to the results of Perret et al. (2019) and Blackman and Perret (2016), respectively. Filled triangles correspond to $\sigma_{u_{\mathrm{L}}}^{2}$ obtained using a frequency cutoff $\left(f_{\mathrm{c}}\right)$ as in Mathis et al. (2009) with $f_{\mathrm{c}}=0.3 U_{\mathrm{e}} / \delta$ applied to the present HWA data. Green open circles are for $\left\langle\overline{u^{\prime} w^{\prime}}\right\rangle$ from Blackman and Perret (2016) 
total velocity variance $\sigma_{u}^{2}$ and the Reynolds shear stress $\left\langle\overline{u^{\prime} w^{\prime}}\right\rangle$ is shown in Fig. 11b. The extracted large scales using the first S-POD modes are found to be contributors to both the Reynolds shear stress and the variance of the streamwise velocity component above the roughness sublayer (i.e. $z \gtrsim 2 h$ ) as already observed in flows over urban canopies (Castro et al. 2006; Coceal et al. 2007). Comparison with the results from Blackman and Perret (2016) and Perret et al. (2019) obtained in the same flow configuration shows a relatively good qualitative agreement given the different methods employed to extract the large-scale component from $u^{\prime}$ in the three studies. Blackman and Perret (2016) indeed used a multi-time delay stochastic estimation-based method to extract $u_{\mathrm{L}}^{\prime}$ from synchronized SPIV and HWA measurements while Perret et al. (2019) employed two-point single HWA measurements to extract the part of $u^{\prime}(z)$ the most correlatedwith $u^{\prime}(z / h=5)$ measured by a fixed reference probe (the reader is referred to their work for a complete description of the corresponding methods and results). For the sake of completeness, the method of Mathis et al. (2009) to extract $u_{\mathrm{L}}$ using a sharp low-pass filter in the spectral domain is applied to the present HWA data (red triangles in Fig. 11b). The cutoff frequency is set to $f_{\mathrm{c}}=0.3 U_{\mathrm{e}} / \delta$ following the analysis of Basley et al. (2018) of the same flow configuration. The overall agreement with the S-POD method is good, in particular for $z / h>2$. Closer to the canopy, the discrepancy is larger, probably due to the presence of canopy-produced energetic coherent structures superimposed on boundary layer large scales (Perret et al. 2019).

The effectiveness of the scale separation obtained presently motivate further investigation to identify the nature of the interactions between the canopy scales and those from the incoming boundary layer, as detailed below.

\subsection{Scale interaction}

An analysis of the interaction of these large-scale structures with the smaller scale turbulence is presented here. The emphasis is on the existence of the non-linear interaction that resembles an amplitude modulation mechanism that has been shown to exist in smooth-wall flow (Mathis et al. 2011) but also in flow over urban-like roughness (Anderson 2016; Basley et al. 2018; Blackman and Perret 2016). The third-order moment of the streamwise velocity component can be written as

$\left.\left\langle\overline{u^{\prime 3}}\right\rangle=\left\langle\overline{u_{\mathrm{L}}^{\prime 3}}\right\rangle+3\left\langle\overline{u_{\mathrm{L}}^{\prime 2} u_{\mathrm{S}}^{\prime}}\right\rangle+3 \overline{\left\langle u_{\mathrm{L}}^{\prime} u_{\mathrm{S}}^{\prime 2}\right.}\right\rangle+\left\langle\overline{u_{\mathrm{S}}^{\prime 3}}\right\rangle$,

where $u_{\mathrm{S}}^{\prime}$ is the small-scale component of $u^{\prime}$ and $u_{\mathrm{L}}^{\prime}$ the largescale component such that $u^{\prime}=u_{\mathrm{L}}^{\prime}+u_{\mathrm{S}}^{\prime}$. Mathis et al. (2011) used the same type of decomposition in their study of a flat plate turbulent boundary layer and showed that the crossterm $3\left\langle\overline{u_{\mathrm{L}}^{\prime} u_{\mathrm{S}}^{\prime 2}}\right\rangle$ is directly linked to the existence of a mechanism of amplitude modulation of the near-wall turbulence by the larger scales. The above results have demonstrated that the present decomposition method (even if based on an energetic criterion) has allowed for the identification of the known VLSMs existing in boundary layer flows. Therefore, $\left\langle\overline{u_{\mathrm{L}}^{\prime} u_{\mathrm{S}}^{\prime 2}}\right\rangle$ can be viewed as the indicator of interaction between the identified structures and the remaining of the flow (of smaller scale). Even if the term $\left\langle\overline{u_{\mathrm{L}}^{\prime} u_{\mathrm{S}}^{\prime 2}}\right\rangle$ does not allow for a precise quantification of the magnitude and the direction of the mean energy transfers, its non-zero value demonstrates the existence of non-linear interactions between scales (Blackman et al. 2018a). The contribution of the largescale component to the skewness of the streamwise velocity component is shown in Fig. 12. While the skewness of the large-scale part $\left\langle\overline{u_{\mathrm{L}}^{\prime 3}}\right\rangle$ is found to be almost zero in the entire domain, the skewness of the small-scale component $\left\langle\overline{u_{\mathrm{S}}^{\prime 3}}\right\rangle$ is, in contrast, a non-negligible contributor to the total skewness of the streamwise velocity fluctuations in the roughness sublayer (i.e. $z / h<3$ ). It is noticeable that the evolution of this term with the wall-normal distance is very similar to the evolution of the skewness of the streamwise velocity across a mixing layer in which positive values are found in the low-speed region and negative values on the high-speed side of the shear-layer (Druault et al. 2005). This may suggest that once the influence of the largest scales is removed, the roughness-sublayer flow behaves as a shear layer flow. This is consistent with what has been observed in boundary layer flows over vegetation canopy (Finnigan 2000). The investigation of this point will be addressed in future work. The terms $\left\langle\overline{u_{\mathrm{L}}^{\prime} u_{\mathrm{S}}^{\prime 2}}\right\rangle$ and $\left.\overline{\left\langle u_{\mathrm{L}}^{\prime 2} u_{\mathrm{S}}^{\prime}\right.}\right\rangle$ representing non-linear interactions between the large and small scales are also found to contribute largely to the skewness. The importance of these two terms indicates that the large and small scales are phasecoupled through a mechanism that resembles an amplitude

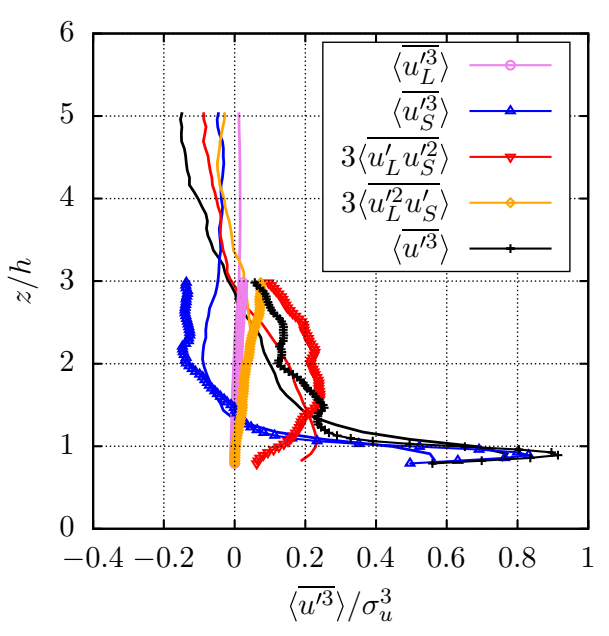

Fig. 12 Scale-decomposed skewness of the streamwise velocity component of (lines) the present study and (symbols) Blackman and Perret (2016) (all terms normalized by $\sigma_{u}^{3 / 2}$ ) 
modulation mechanism as found by Mathis et al. (2009) in smooth-wall boundary layers. For the sake of completeness, the present results are compared with those from Blackman and Perret (2016) obtained in the same flow configuration (symbols in Fig. 12). As mentioned above, given the difference between the employed methods to extract the large scales from the raw $u^{\prime}$ component and the extent of the SPIV measurement regions, the agreement is fairly good, demonstrating the relevance of the present S-POD approach to perform scale decomposition and to identify scale-interaction mechanisms.

Finally, the spectral decomposition of the term $\left\langle\overline{u_{\mathrm{L}}^{\prime} u_{\mathrm{S}}^{\prime 2}}\right\rangle$, which bears the footprint of the amplitude modulation mechanism is analyzed. The present S-POD analysis gives access to time-resolved large-scale signals and, therefore, allows for cross-spectral analysis of $u_{\mathrm{L}}^{\prime}$ and $u_{\mathrm{S}}^{\prime 2}$. Computing the spectral coherence between these two signals is equivalent to the analysis as a function of frequency of the correlation coefficient between $u_{\mathrm{L}}^{\prime}$ and the large-scale envelope of $u_{\mathrm{S}}^{\prime}$. The coherence map between $u_{\mathrm{L}}^{\prime}$ and $u_{\mathrm{S}}^{\prime 2}$ is shown in Fig. 13 as a function of both the frequency and the wall-normal distance $z$. While the coherence level is below $\approx 0.1$, a maximum is manifest for $z / h \approx 1$ and $f \delta / \bar{u}(z) \approx 0.15$ indicating that the large scales modulate the large-scale component of the envelope of the small-scale time history as also found by Mathis et al. (2009) for smooth-wall configurations. While not reported here for conciseness, the phase of the cross-spectrum is non-zero. This confirms that the maximum of amplitude modulation occurs with a time delay between the large and the small scales, in agreement with the finding of Blackman and Perret (2016) and Guala et al. (2011), for instance. In addition, it must be noted that when the interaction between $u_{\mathrm{L}}$ and the spanwise or the wall-normal components $v$ or $w$, respectively, is considered (Fig. 14), the profiles of $3\left\langle\overline{u_{\mathrm{L}}^{\prime} u_{j}^{\prime 2}}\right\rangle, j=2$ or 3 , are qualitatively similar to that of

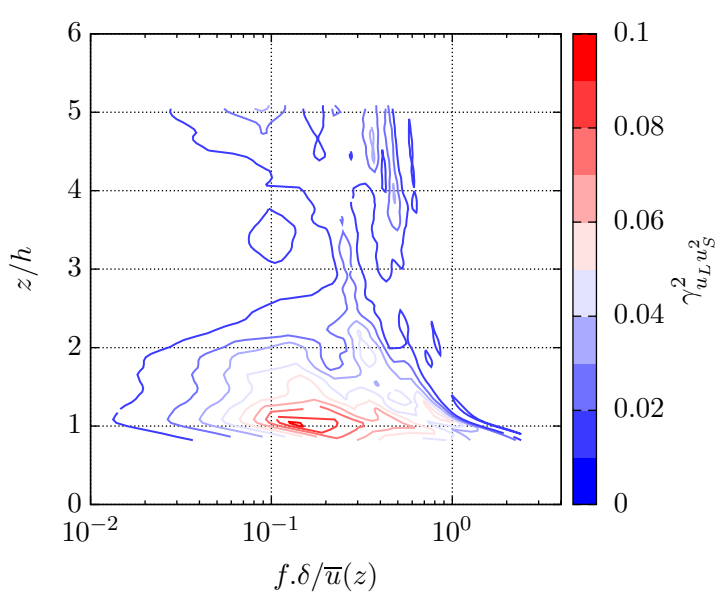

Fig. 13 Spectral coherence between the large scales $u_{\mathrm{L}}^{\prime}$ and $u_{\mathrm{S}}^{\prime 2}$ as a function of the wall-normal location

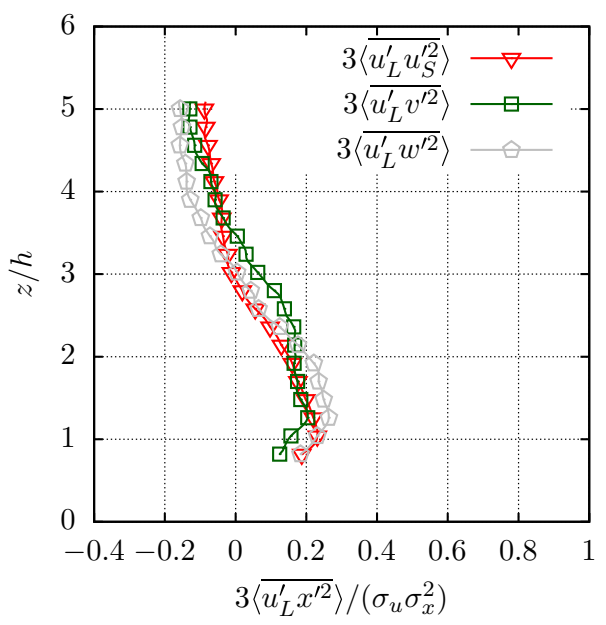

Fig. 14 Third-order moments $3\left\langle\overline{u_{\mathrm{L}}^{\prime} u_{\mathrm{S}}^{\prime 2}}\right\rangle, 3\left\langle\overline{u_{\mathrm{L}}^{\prime} v^{\prime 2}}\right\rangle$ and $3\left\langle\overline{u_{\mathrm{L}}^{\prime} w^{\prime 2}}\right\rangle$ (all terms normalized by $\left.\sigma_{u} \sigma_{\{u, v, w\}}^{2}\right\}$

$3\left\langle\overline{u_{\mathrm{L}}^{\prime} u_{\mathrm{S}}^{\prime 2}}\right\rangle$. Even if the scales of $w$ and $v$ involved in these cross-terms remain to be identified, it suggests that in the roughness sublayer, the low- and high-speed structures of largest scales interact with structures involving the three components of the velocity through the same type of nonlinear mechanisms.

\section{Conclusions}

A high-Reynolds number boundary layer flow developing over large roughness elements representative of the urban canopy has been investigated via the use of the recently introduced spectral POD (Sieber et al. 2016). It has been demonstrated that this approach, which acts as a band-pass filtering operation on the temporal coefficients obtained from the conventional snapshot POD, enables to account for the long-term coherence of the most energetic largescale structures existing in the outer layer of the boundary layer. When applied to stereoscopic PIV velocity fields that are of limited extent in the streamwise direction and which have been acquired with a sampling frequency too low to properly capture the spectral content of the flow, S-POD proved to be able to extract the large-scale coherent structures from the flow via the proper choice of the filter width. In the present case, the use of the S-POD can be viewed as augmenting the snapshot POD kernel by extending the database in the streamwise direction through the use of Taylor's approximation. This case would correspond to the use of a simple low-pass box filter. A modification of the definition of the spatial S-POD modes has been proposed so that they are orthonormal to each other. 
It results in spatio-temporal modes which reflect the statistical organization of the most energetic structures both in space and time.

The characteristics of the educed large-scale coherent structures have been shown to correspond well to that of the VLSMs identified in the turbulent boundary layer in the literature. In agreement with previous work in similar flow configurations, the large scales extracted via S-POD have been found to consist of elongated low- or high-momentum regions. They are major contributors to the turbulent kinetic energy and the Reynolds shear stress in the outer region of the flow. The extracted large-scale structures were shown to interact with small-scale near-wall turbulence through a mechanism of both superimposition and amplitude modulation. The three velocity components were found to be under the influence of the large-scale component of the streamwise velocity component, in agreement with recent findings in smooth- and rough-wall boundary layers (Talluru et al. 2014; Blackman and Perret 2016).

Acknowledgements The authors would like to thank Dr. Cédric Rivet for providing the SPIV data and Mr Thibaut Piquet for his technical support during the experimental program. The authors also acknowledge the financial support of the French National Research Agency through the research Grant URBANTURB no. ANR-14-CE22-0012-01.

\section{References}

Adrian RJ (2007) Hairpin vortex organization in wall turbulence. Phys Fluids 19(4):041301. https://doi.org/10.1063/1.2717527

Anderson W (2016) Amplitude modulation of streamwise velocity fluctuations in the roughness sublayer: evidence from large-eddy simulations. J Fluid Mech 789:567-588

Basley J, Perret L, Mathis R (2018) Spatial modulations of kinetic energy in the roughness sublayer. J Fluid Mech 850:584-610

Blackman K, Perret L (2016) Non-linear interactions in a boundary layer developing over an array of cubes using stochastic estimation. Phys Fluids 28(095):108

Blackman K, Perret L, Calmet I, Rivet C (2017) Turbulent kinetic energy budget in the boundary layer developing over an urban-like rough wall using PIV. Phys Fluids 29(085):113

Blackman K, Perret L, Calmet I (2018a) Energy transfer and non-linear interactions in an urban boundary layer using stochastic estimation. J Turb 19(10):849-867

Blackman K, Perret L, Savory E (2018b) Effect of upstream flow regime and canyon aspect ratio on non-linear interactions between a street canyon flow and the overlying boundary layer. BoundLayer Meteorol 169(3):537-558

Calluaud D, David L (2004) Stereoscopic particle image velocimetry measurements of the flow around a surface-mounted block. Exp Fluids 36(1):53-61

Castro IP (2007) Rough-wall boundary layers: mean flow universality. J Fluid Mech 585:469-485

Castro IP, Cheng H, Reynolds R (2006) Turbulence over urban-type roughness: deductions from wind-tunnel measurements. BoundLayer Meteorol 118:109-131

Cheng H, Hayden P, Robins AG (2007) Flow over cube arrays of different packing densities. J Wind Eng Ind Aerodyn 95:715-740
Coceal O, Dobre A, Thomas TG (2007) Unsteady dynamics and organized structures from DNS over an idealized building canopy. Int J Climatol 27:1943-1953

del Alamo JC, Jimenez J (2003) Spectra of the very large anisotropic scales in turbulent channels. Phys Fluids 15:41-44

Dennis DJC, Nickels TB (2008) On the limitations of taylor's hypothesis in constructing long structures in a turbulent boundary layer. J Fluid Mech 614:197-206. https://doi.org/10.1017/S002211200 8003352

Druault P, Delville J, Bonnet J (2005) Experimental 3D analysis of the large scale behaviour of a plane turbulent mixing layer. Flow Turbul Combust 74(2):207-233

Finnigan JJ (2000) Turbulence in plant canopies. Annu Rev Fluid Mech 32:519-571

Grimmond CSB, Oke TR (1999) Aerodynamic properties of urban areas derived from analysis of surface form. J Appl Meteorol 38:1262-1292

Guala M, Metzger M, McKeon BJ (2011) Interactions within the turbulent boundary layer at high reynolds number. J Fluid Mech 666:573-604

Herpin S, Perret L, Mathis R, Tanguy C, Lasserre JJ (2018) Investigation of the flow inside an urban canopy immersed into an atmospheric boundary layer using Laser Doppler anemometry. Exp Fluids 59:80-103

Hultmark M, Smits AJ (2010) Temperature corrections for constant temperature and constant current hot-wire anemometers. Meas Sci Technol 21:1-4

Hutchins N, Marusic I (2007) Evidence of very long meandering features in the logarithmic region of turbulent boundary layers. J Fluid Mech 579:1-28

Hutchins N, Chauhan K, Marusic I, Monty J, Klewicki J (2012) Towards reconciling the large-scale structure of turbulent boundary layers in the atmosphere and laboratory. Bound-Layer Meteorol 145(2):273-306

Inagaki A, Kanda M (2010) Organized structure of active turbulence over an array of cubes within the logarithmic layer of atmospheric flow. Bound-Layer Meteorol 135:209-228

Jackson PS (1981) On the displacement height in the logarithmic velocity profile. J Fluid Mech 111:15-25

Jimenez J (2004) Turbulent flows over rough walls. Annu Rev Fluid Mech 36:173-196

Lumley JL (1967) The structure of inhomogeneous turbulent flows. In: Yaglom AM, Tatarski VI (eds) Atmospheric turbulence and radio propagation. Nauka, Moscow, pp 166-178

Marusic I, Hutchins N (2008) Study of the log-layer structure in wall turbulence over a very large range of Reynolds number. Flow Turbul Combust 81:115-130

Mathis R, Hutchins N, Marusic I (2009) Large-scale amplitude modulation of the small-scale structures in turbulent boundary layers. J Fluid Mech 628:311-337

Mathis R, Hutchins N, Marusic I, Sreenivasan KR (2011) The relationship between the velocity skewness and the amplitude modulation of the small scale by the large scale in turbulent boundary layers. Phys Fluids 23:1-4

Mendez MA, Balabane M, Buchlin JM (2018) Multi-scale proper orthogonal decomposition of complex fluid flows. arXiv e-prints arXiv:1804.09646, arXiv:1804.09646

Monty JP, Stewart JA, Williams RC, Chong MS (2007) Large-scale features in turbulent pipe and channel flows. J Fluid Mech 589:147-156

Perret L, Rivet C (2018) A priori analysis of the performance of cross hot-wire probes in a rough wall boundary layer based on stereoscopic PIV. Exp Fluids 59(10):153

Perret L, Savory E (2013) Large-scale structures over a single street canyon immersed in an urban-type boundary layer. Bound-Layer Meteorol 148:111-131 
Perret L, Basley J, Mathis R, Piquet T (2019) The atmospheric boundary layer over urban-like terrain: Influence of the plan density on roughness sublayer dynamics. Bound-Layer Meteorol 170(2):205234. https://doi.org/10.1007/s10546-018-0396-9

Placidi M, Ganapathisubramani B (2015) Effects of frontal and plan solidities on aerodynamic parameters and the roughness sublayer in turbulent boundary layers. J Fluid Mech 782:541-566

Rivet C, Perret L, Rosant JM (2012) Scale interactions in an urbanlike boundary layer: a wind tunnel study. In: 20th Symposium on boundary layers and turbulence, Boston, MA

Savory E, Perret L, Rivet C (2013) Modeling considerations for examining the mean and unsteady flow in a simple urban-type street canyon. Meteorol Atmos Phys 121:1-16

Sieber M, Paschereit CO, Oberleithner K (2016) Spectral proper orthogonal decomposition. J Fluid Mech 792:798-828

Sirovich L (1987) Turbulence and the dynamics of coherent structures. Part I: coherent structures. Q Appl Math 45(3):561-571

Snyder WH, Castro IP (2002) The critical Reynolds number for roughwall boundary layers. J Wind Eng Ind Aerodyn 90:41-54

Soloff SM, Adrian RJ, Liu ZC (1997) Distortion compensation for generalized stereoscopic particle image velocimetry. Meas Sci Technol 8(12):1441-1454
Takimoto H, Sato A, Barlow JF, Moriwaki R, Inagaki A, Onomura S, Kanda M (2011) Particle image velocimetry measurements of turbulent flow within outdoor and indoor urban scale models and flushing motions in urban canopy layers. Bound-Layer Meteorol 140:295-314

Talluru KM, Baidya R, Hutchins N, Marusic I (2014) Amplitude modulation of all three velocity components in turbulent boundary layers. J Fluid Mech 746:R1. https://doi.org/10.1017/jfm.2014.132

Towne A, Schmidt OT, Colonius T (2018) Spectral proper orthogonal decomposition and its relationship to dynamic mode decomposition and resolvent analysis. J Fluid Mech 847:821-867

Volino RJ, Schultz MP, Flack KA (2011) Turbulence structure in boundary layers over periodic two- and three-dimensional roughness. J Fluid Mech 676:172-190 